\title{
Lesson of the month 1: Obesity hypoventilation (Pickwickian) syndrome: a reversible cause of severe pulmonary hypertension
}

\author{
Authors: Frazer Warricker, ${ }^{A}$ Zafir Islam ${ }^{B}$ and Benoy N Shah ${ }^{C}$
}

\begin{abstract}
Obesity hypoventilation syndrome (OHS) is a condition in which an individual with a body mass index $>30 \mathrm{~kg} / \mathrm{m}^{2}$ develops daytime alveolar hypoventilation (defined as a resting $\mathrm{PaCO}_{2}$ $>45 \mathrm{mmHg}$ ) that cannot be attributed to other pathologies. It is a condition with increasing prevalence and rising cost to healthcare systems worldwide. Right heart failure and pulmonary hypertension are well-known complications of this syndrome. Here, we present the case of a female patient with $\mathrm{OHS}$ who presented to our centre with severe pulmonary hypertension, which resolved with appropriate treatment. We also review this clinical condition and its diagnosis and management.
\end{abstract}

KEYWORDS: obesity hypoventilation syndrome, Pickwickian syndrome, pulmonary hypertension, obstructive sleep apnoea

\section{Introduction}

Obesity hypoventilation syndrome (OHS), also known as Pickwickian Syndrome, is an under-recognised condition that can cause respiratory failure, right heart failure and severe pulmonary hypertension $(\mathrm{PH})$. Individuals with $\mathrm{OHS}$ develop daytime alveolar hypoventilation, resulting in hypercapnia and hypoxaemia, which cannot be attributed to other pathologies (Table 1). ${ }^{1}$ Of patients with OHS, $90 \%$ also have obstructive sleep apnoea (OSA), while the remaining 10\% experience nocturnal hypoventilation but without frequent episodes of apnoea. ${ }^{2}$ In addition to respiratory impairment, OHS is also a known cause of PH (Table 2).

In recent years, the prevalence of OHS has markedly increased; it is estimated that $0.3-0.4 \%$ of the general population and up to $30 \%$ of inpatients with a body mass index (BMI) $>35 \mathrm{~kg} / \mathrm{m}^{2}$ might be affected. ${ }^{3}$ Here, we present a recent case from our institute in which a young patient presenting with breathlessness was found to have severe $\mathrm{PH}$ and respiratory failure; after receiving a diagnosis of $\mathrm{OHS}$ and appropriate management, the patient now is well with no $\mathrm{PH}$.

Authors: ${ }^{A}$ CT2 Medicine, Department of Cardiology, University Hospital Southampton, Southampton, UK; ${ }^{B}$ CT1 Medicine, Department of Cardiology, University Hospital Southampton, Southampton, UK; ' Consultant cardiologist, Department of Cardiology, University Hospital Southampton, Southampton, UK
Table 1. Diagnostic criteria for obesity

hypoventilation syndrome

Criteria

Description

Obesity

Body mass index $>30 \mathrm{~kg} / \mathrm{m}^{2}$

Hypoventilation Awake arterial hypercapnia $\left(\mathrm{PaCO}_{2}>45 \mathrm{mmHg}\right)$

Sleep-disordered Polysomnography reveals sleep

breathing hypoventilation with nocturnal hypercapnia with or without obstructive apnea/hypopnea events

Rule out other causes of Severe chronic obstructive pulmonary disease, severe interstitial lung disease, chest wall hypoventilation disorders, thyroid disorders and congenital hypoventilation syndromes

\section{Lesson}

A 39-year-old Fijian woman presented to the cardiology clinic with a chronic history of exertional dyspnoea, with an acute worsening over the past 3 months. Her exercise tolerance was 50 yards (approximately $46 \mathrm{~m}$ ). She denied chest pains or palpitations but complained of disturbed sleep and of having to sleep in a chair. Her family also mentioned loud snoring at night and daytime somnolence.

On examination, her weight and height were $132 \mathrm{~kg}$ and $167 \mathrm{~cm}$, respectively (BMI $47 \mathrm{~kg} / \mathrm{m}^{2}$ ). Her resting blood pressure was $123 / 75 \mathrm{mmHg}$, her pulse was 100 beats per minute, with a resting oxygen saturation of $70 \%$ breathing room air. She had no audible murmurs but did have a raised jugular venous pressure (JVP) with bilateral pitting oedema. She mobilised no more than $10 \mathrm{~m}$ to undergo electrocardiography (ECG; which revealed sinus tachycardia with partial right bundle branch block) and became acutely more breathless, with her oxygen saturation dropping to $60 \%$. An urgent arterial blood gas sample revealed type 2 respiratory failure ( $\mathrm{pH} 7.41$, $\mathrm{PaCO}_{2} 7.8 \mathrm{kPa}, \mathrm{PaO}_{2} 4.2 \mathrm{kPa}$ and $\mathrm{HCO}_{3}{ }^{-} 36.6 \mathrm{mmol} / \mathrm{L}$ ) with a markedly elevated bicarbonate level, implying chronic $\mathrm{CO}_{2}$ retention. Transthoracic echocardiography (TTE) revealed an enlarged right heart with an estimated pulmonary arterial systolic pressure $>90 \mathrm{mmHg}$, consistent with severe $\mathrm{PH}$.

She was urgently admitted to the coronary care unit and treated with intravenous diuretics and low-flow oxygen. Unfortunately, her condition deteriorated, requiring transfer to the intensive care unit for non-invasive ventilation (NIV). 
Table 2. Clinical classification of pulmonary

hypertension

\section{Type of pulmonary hypertension}

Grade 1

Pulmonary arterial hypertension

Pulmonary venoocclusive disease and/ or pulmonary capillary haemangiomatosis

\section{Grade 2}

Pulmonary hypertension resulting from left heart disease

\section{Grade 3}

Pulmonary hypertension resulting from lung diseases and/or hypoxia

Grade 4

Chronic thromboembolic pulmonary hypertension

Grade 5

Pulmonary hypertension with unclear and/ or multifactorial mechanisms

$\mathrm{ALK}=$ anaplastic lymphoma kinase; $\mathrm{BMPR}=$ bone morphogenetic protein receptor; $\mathrm{LV}=$ left ventricular

Idiopathic

Drugs and toxins

Connective tissue diseases

HIV infection

Portal hypertension

Congenital heart disease

Schistosomiasis the newborn

LV systolic dysfunction

LV diastolic dysfunction

Valvular heart disease Interstitial lung disease

Sleep-disordered breathing

Alveolar hypoventilation disorders

Chronic exposure to high altitude

Developmental abnormalities

Haematological disorders (eg Systemic disorders (eg sarcoidosis, lymphangioleiomyomatosis, neurofibromatosis, vasculitis)

Metabolic disorders (eg glycogen thyroid disorders) mediastinitis, chronic renal failure
Heritable: (eg BMPR, ALK1, mutations)

Persistent pulmonary hypertension of

Chronic obstructive pulmonary disease

Other pulmonary diseases with mixed restrictive and obstructive patterns myeloproliferative disorders, splenectomy) pulmonary Langerhans cell histiocytosis, storage disease, Gaucher's disease,

Others: tumoural obstruction, fibrosing
Urgent CT pulmonary angiography revealed neither acute nor chronic pulmonary emboli.

Following bi-level positive airways pressure (BiPAP) ventilation, repeat echocardiography $24 \mathrm{~h}$ after admission showed a reduction in her estimated right heart pressures from $90 \mathrm{mmHg}$ to $60 \mathrm{mmHg}$. Given that the patient's pulmonary pressures had improved with oxygen, it was felt that idiopathic $\mathrm{PH}$ was unlikely. A clinical diagnosis of $\mathrm{OHS}$ with chronic with respiratory support and diuretics, and she made an excellent recovery, being discharged home 13 days after before discharge showed further reduction of her right heart pressure to $31 \mathrm{mmHg}$. She was seen in the outpatient clinic 4 months later, at which point she appeared very well and had no symptoms. Repeat echocardiography now showed undetectable right heart pressures, indicating normalisation of the pulmonary pressures (Fig 1). She continues with nocturnal NIV and no longer has poor sleep or daytime somnolence.

\section{Discussion}

OHS was first described in 1956, at which time the condition was referred to as 'Pickwickian syndrome' after the character Joe, an overweight red-faced boy in the novel The Pickwick Papers by Charles Dickens. ${ }^{4} \mathrm{OHS}$ can present with a range of symptoms, from complaints of snoring and daytime somnolence by family members to breathlessness, fatigue, mood disorders and morning headaches. The past medical history can reveal conditions that provide alternative explanations for $\mathrm{CO}_{2}$ retention, including mechanical disorders, such as lung diseases and kyphoscoliosis, neuropathies, and central nervous system abnormalities, such as prior stroke and severe hypothyroidism. A careful social history is required to screen for potential respiratory suppressants, such as alcohol and narcotic drugs. Physical examination invariably reveals significant obesity and frequently an elevated resting respiratory rate, and can show features of right heart failure, such as peripheral oedema. Several initial investigations are warranted; a full blood count can reveal erythrocytosis and thyroid function should also be checked. ECG frequently reveals sinus tachycardia, can show right bundle branch block, and often right heart abnormalities, including elevated pulmonary arterial pressures. Arterial blood gas analysis should be performed and usually reveals a compensated respiratory acidosis, with elevated $\mathrm{PaCO}_{2}$, reduced $\mathrm{PaO}_{2}$ and elevated serum bicarbonate levels. $\mathrm{OHS}$ is associated with Grade 3 pulmonary hypertension. ${ }^{7}$ When diagnosing $\mathrm{PH}$, the gold standard investigation is right heart catheterisation (RHC); however, TTE is commonly used for screening and monitoring of the disease because it is non-invasive, cheaper, more widely available and has good diagnostic accuracy. ${ }^{8}$

Treatment aims for patients with OHS are based on minimising morbidity and mortality by normalising arterial $\mathrm{CO}_{2}$ levels. Other goals include the prevention of oxygen desaturation, reversal of erythrocytosis, cor pulmonale and symptomatic relief of daytime somnolence. ${ }^{9}$ These goals are achieved through a multidisciplinary approach, with the mainstay treatment being long-term lifestyle changes focused on weight loss. ${ }^{10}$ Weight loss of at least $10 \mathrm{~kg}$ results in a significant improvement in vital capacity and maximum voluntary ventilation and a significant reduction in daytime (untreated) OSA was made. The patient's symptoms improved admission with nocturnal NIV. Repeat echocardiography 

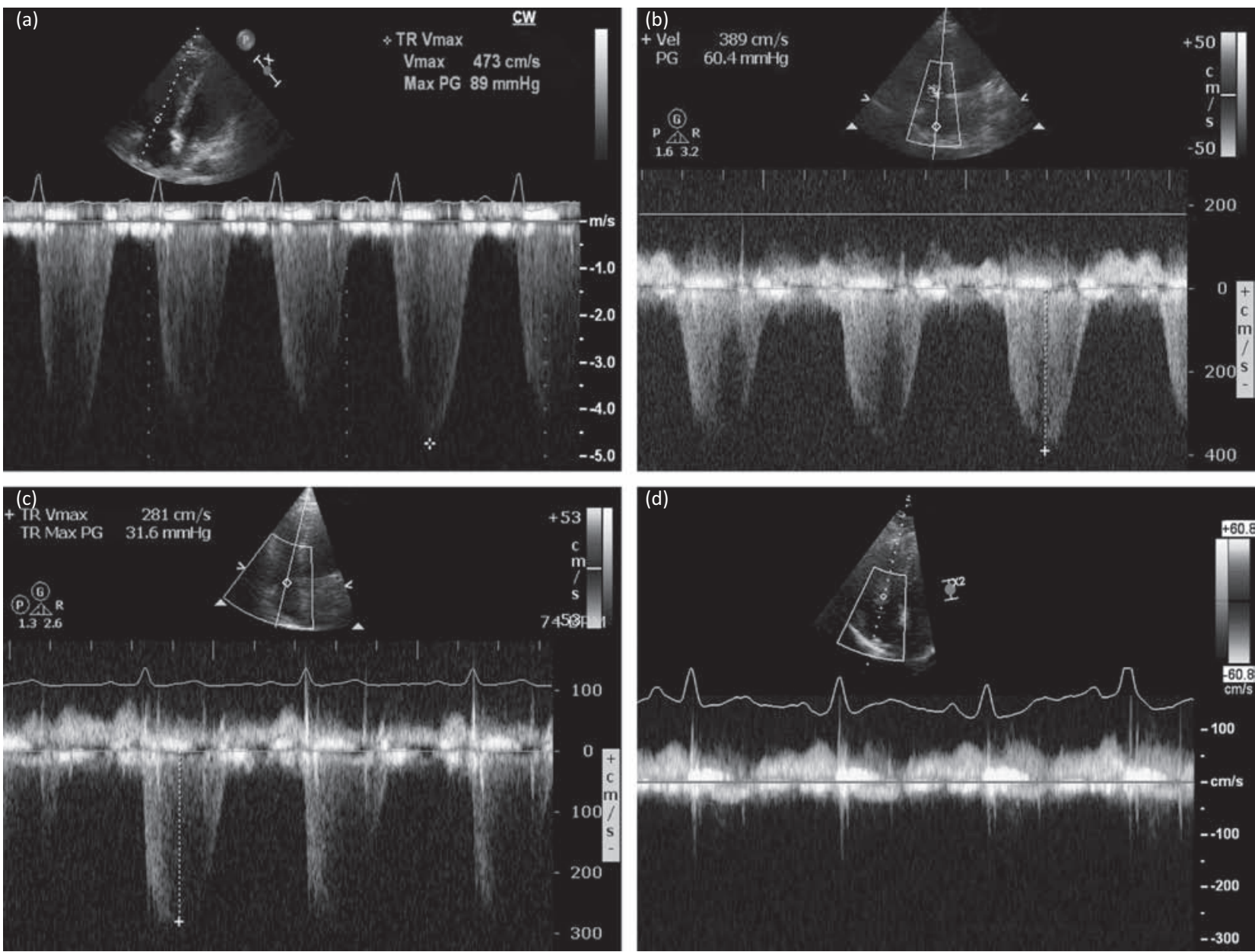

Fig 1. Doppler echocardiographic recordings taken across the tricuspid valve, using the velocity of the tricuspid regurgitant (TR) jet to estimate right ventricular systolic pressure (RVSP). On first presentation, a severely elevated RVSP of $89 \mathrm{mmHg}+$ right atrial (RA) pressure was recorded (a). After $24 \mathrm{~h}$ of treatment, this improved to $60 \mathrm{mmHg}+\mathrm{RA}$ pressure (b). Before discharge from hospital, the RVSP had fallen further to $31 \mathrm{mmHg}+\mathrm{RA} \mathrm{pressure} \mathrm{(c)}$ and, when the patient was seen several months after discharge, there was no detectable TR jet and no evidence of pulmonary hypertension (d).

$\mathrm{PaCO}_{2}$. In cases where lifestyle changes prove to be ineffective or if the patient fails to tolerate NIV, bariatric surgery has been considered. ${ }^{2,9}$ A randomised control trial evaluating the treatment impact between BiPAP and continuous positive airway pressure (CPAP) showed that, after 3 months, both methods resulted in improvements in symptoms, quality of life and respiratory failure, with no statistically significant difference between the two. ${ }^{8}$

\section{Conclusion}

With an increasing prevalence of OHS, it is important that medical professionals both in primary and secondary care are aware of this obesity-related condition. With aggressive early lifestyle intervention, it might be possible to avoid the development of OHS and related $\mathrm{PH}$ in a bid to reduce hospitalisation rates, morbidity and mortality.

\section{Conflicts of interest}

The authors have no conflicts of interest to declare.

\section{Author contributions}

FW and ZI wrote the first draft of the manuscript. BNS revised and critically appraised the manuscript. BNS is the guarantor.

\section{References}

1 Kaw R. Obesity and pulmonary hypertension. What's the link? $\mathrm{Br} J$ Med Pract 2009;2:4-5.

2 Egea-Santaolalla C, Javaheri S. Obesity hypoventilation syndrome. Curr Sleep Med Rep 2016;2:12-9.

3 Hart N, Mandal S, Manuel A et al. Obesity hypoventilation syndrome: does the current definition need revisiting? Thorax 2014;69:83-4.

4 Olsen A, Zwillich C. The obesity hypoventilation syndrome. Am J Med 2005;118:948-56.

5 Tulaimat A, Littleton S. Defining obesity hypoventilation syndrome. Thorax 2014;69:491.

6 Naeije R. Pulmonary hypertension in hypoventilation syndromes. Eur Resp J 2014;43:12-5.

7 Galie N, Humbert M, Vachiery J-L et al. 2015 ESC/ERS Guidelines for the diagnosis and treatment of pulmonary hypertension. Eur Heart J 2016;37:67-119. 
8 Kauppert C A, Dvorak I, Kollert F et al. Pulmonary Hypertension in obesity-hypoventilation syndrome. Resp Med 2013;107:2061-70.

9 Brown LK. Obesity hypoventilation syndrome. Curr Sleep Med Rep 2015;1:241-50.

10 Nolte JES, Koehler U, Sohrabi AK et al. Reversible severe pulmonary hypertension in obesity hypoventilation and Mohr Syndrome. Resp Med 2011;4:30-2.
Address for correspondence: Benoy N Shah, Cardiac Administration Offices, Level E North Wing, Southampton General Hospital, Tremona Road, Southampton S016 6YD, UK.

Email: benoy.shah@uhs.nhs.uk

\title{
Lesson of the month 2: Use of thrombolysis for ischaemic stroke in pregnancy - a case report and review of literature
}

\author{
Authors: Asim Khan, ${ }^{A}$ Paris Hosseini, ${ }^{B}$ Branimir Nevajda ${ }^{C}$ and Sami Khan ${ }^{D}$
}

A nine-week pregnant 33-year-old female presented with sudden-onset right-sided hemiparesis, hemisensory loss, dysarthria and homonymous hemianopia. She was known to have eleven previous miscarriages and used recreational drugs. A CT-head was unremarkable. The patient was subsequently diagnosed with an acute thromboembolic infarct and chose alteplase after counselling. During thrombolysis an ultrasound identified a foetus with no concerning features.

A post-thrombolysis $C T$ revealed a left-sided posterior cerebral infarct. CT-venography, carotid Doppler, 72-hour Holter monitor, thrombophilia and an autoimmune screen were all normal. A transthoracic echocardiogram demonstrated a mobile intra-atrial septum with a patent foramen ovale confirmed on bubble echocardiogram. Three days postthrombolysis the patient requested a termination of pregnancy. A subsequent transvaginal ultrasound demonstrated a missed miscarriage for which the patient underwent evacuation of retained products of conception. Multidisciplinary care ensured that her sensorimotor deficit resolved grossly with only mild dysarthria and right-hand fine motor incoordination.

\section{Case history}

A 33-year-old female in the ninth week of gestation presented with sudden-onset right-sided weakness, numbness and speech difficulty. Her previous medical history included eleven

Authors: ${ }^{\mathrm{A}}$ core medical trainee, Basildon University Hospital, Basildon and Thurrock NHS Foundation Trust; ${ }^{\mathrm{B}}$ medical student, University College London Medical School, University College London; ' neurology and stroke consultant, Basildon University Hospital, Basildon and Thurrock NHS Foundation Trust; ${ }^{D}$ radiology consultant, Basildon University Hospital, Basildon and Thurrock NHS Foundation Trust

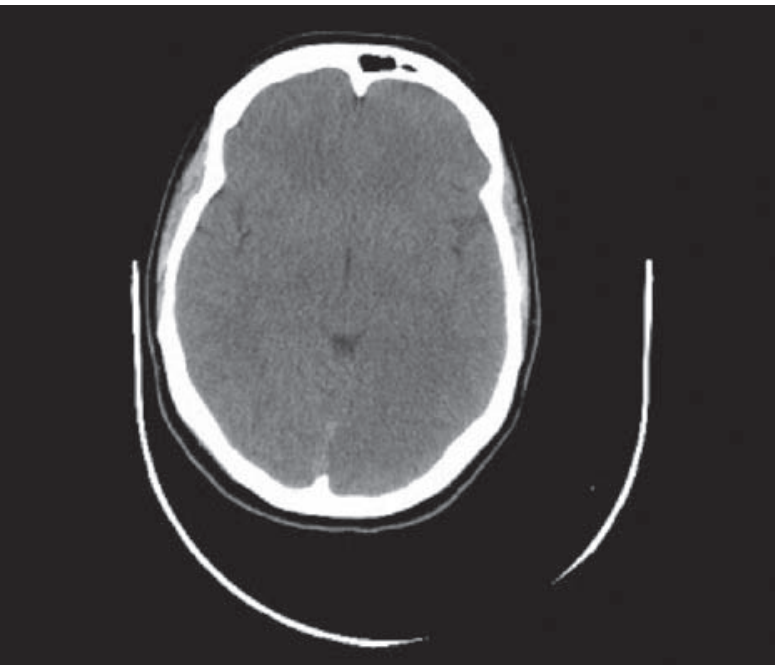

Fig 1. Initial CT-head pre-thrombolysis. Axial view. Upon retrospective analysis there is subtle evidence of the posterior cerebral infarct.

miscarriages of unknown cause. She had a ten-pack year smoking history and also used cocaine, cannabis, heroin and amphetamine recreationally.

Her vital observations and capillary blood glucose were normal. She was not confused. Speech assessment revealed dysarthria. Cranial nerve examination demonstrated a rightsided facial nerve palsy and homonymous hemianopia. Motor function in the limbs confirmed right-sided hemiparesis with a MRC grade of four and two in the upper and lower limbs respectively. Gait assessment was not possible. Tone was flaccid and reflexes were absent on the right-side with an equivocal right plantar response. There was a sensory deficit on the right-side. Cerebellar assessment demonstrated incoordination. 